\title{
Sinais, sintomas e função vocal em indivíduos com disfagia tratados de câncer de cabeça e pescoço
}

\author{
Signs, symptoms and vocal function in individuals with dysphagia \\ treated for head and neck cancer
}

Felipe Moreti', Bruna Morasco-Geraldini', Simone Aparecida Claudino-Lopes ${ }^{1}$, Elisabete Carrara-de Angelis ${ }^{1}$

\begin{abstract}
RESUMO
Objetivos: Avaliar a presença de sinais e sintomas vocais e função vocal em indivíduos com disfagia, tratados de câncer de cabeça e pescoço - $\mathrm{CCP}$ e identificar os valores de corte dos respectivos instrumentos. Métodos: Estudo prospectivo com 96 indivíduos ( 68 homens e 28 mulheres), divididos em grupo experimental - GE (pacientes tratados de CCP com disfagia, independente de queixa vocal) e grupo controle - GC (sem queixas autorrelatadas de voz e deglutição, pareados em média de idade e sexo com GE), que responderam a um questionário com dados de identificação e caracterização da amostra, Lista de Sinais e Sintomas Vocais - LSS, e dois protocolos de avaliação vocal (Índice de Função Glótica - IFG e Escala de Sintomas Vocais - ESV). Por fim, as notas de corte dos protocolos foram identificadas por meio da curva ROC (Receiver Operating Characteristic). Resultados: Indivíduos do GE apresentaram mais sinais e sintomas vocais e maiores escores no IFG e ESV que os indivíduos do GC, além de pior autoavaliação vocal. Os três instrumentos apresentaram máxima área sob a curva ROC, com valores de corte $\mathrm{ESV}=17, \mathrm{IFG}=4$ e LSS=6 pontos. Conclusão: Indivíduos tratados de CCP com disfagia apresentam mais sinais e sintomas vocais, pior autopercepção da disfunção vocal e maior prejuízo nos aspectos de limitação, emocional e físico da voz, que indivíduos vocalmente saudáveis. Os três instrumentos evidenciaram sensibilidade e especificidade máximas, podendo ser utilizados como ferramentas de triagem.
\end{abstract}

Palavras-chave: Disfonia; Transtornos de deglutição; Neoplasias de cabeça e pescoço; Fluoroscopia; Inquéritos e questionários; Fonoaudiologia

\begin{abstract}
Purpose: To evaluate the presence of vocal signs and symptoms and the vocal function in patients with dysphagia treated for head and neck cancer-HNC and identify the cutoff values of these instruments. Methods: Prospective study with 96 individuals ( 68 men and 28 women) divided into an experimental group - EG (HNC patients with dysphagia, independently of their vocal complaints) and control group - CG (without self-reported vocal or swallowing complaints, with age and sex-matched to the EG). They all answered a questionnaire with identification and characterization of the sample data, the Vocal Signs and Symptoms List - SSL and the protocols: Glottal Function Index - GFI and Voice Symptom Scale - VoiSS. The protocols cutoff values were identified by the ROC curve. Results: Individuals from the EG had more vocal signs and symptoms and higher scores in the GFI and the VoiSS than individuals from the CG, they also had worst vocal self-assessment. The three instruments showed maximum area under the ROC curve, with cutoff values of VoiSS $=17, \mathrm{GFI}=4$ and $\mathrm{SSL}=6$. Conclusions: Individuals treated for $\mathrm{HNC}$ with dysphagia presented more vocal signs and symptoms, poorer perception of their vocal dysfunction and greater loss in vocal aspects of impairment, emotional and physical than the vocally healthy individuals. The three instruments showed maximum sensitivity and specificity and can be used as screening tools.
\end{abstract}

Keywords: Dysphonia; Deglutition disorders; Head and neck neoplasms; Fluoroscopy; Surveys and questionnaires; Speech, language and hearing sciences

Pesquisa desenvolvida no Departamento de Fonoaudiologia, Centro de Tratamento e Pesquisa do A. C. Camargo Cancer Center / Fundação Antônio Prudente FAP - São Paulo (SP) - Brasil, como pré-requisito para conclusão do Curso de Especialização em Motricidade Oral em Oncologia.

${ }^{1}$ Departamento de Fonoaudiologia, Centro de Tratamento e Pesquisa, Centro Integrado de Diagnóstico, Tratamento, Ensino e Pesquisa - A. C. Camargo Cancer

Center, Fundação Antônio Prudente - FAP - São Paulo (SP), Brasil.

Conflito de interesses: Não.

Contribuição dos autores: FM foi responsável pelo delineamento do estudo, coleta da amostra, escrita e revisão final do manuscrito; BMG foi responsável pela coleta da amostra e revisão do manuscrito; SACL foi coorientadora, responsável pelo delineamento do estudo e revisão final do manuscrito; ECA foi orientadora, responsável pelo delineamento do estudo e revisão final do manuscrito.

Financiamento: Nada a declarar.

Autor correspondente: Felipe Moreti. E-mail: felipemoreti@uol.com.br

Recebido: Abril 21, 2017; Aceito: Maio 22, 2018 


\section{INTRODUÇÃO}

Voz e deglutição possuem uma relação muito próxima, pelo fato de compartilharem várias estruturas anatômicas e neurais para seus processos distintos, como os pares cranianos V, VII, IX, X, XI e XII, que atuam diretamente sobre as funções da fala e da deglutição $0^{(1)}$ e, quando afetados, podem interferir na qualidade de vida do paciente, comprometendo, principalmente, os aspectos social, nutricional e pulmonar ${ }^{(2)}$. Pode ser relativamente comum que pacientes com queixas de voz apresentem sintomas aerodigestivos e pacientes com queixas de deglutição apresentem sintomas vocais, sendo foco de terapia a voz e a deglutição, com diferentes abordagens e evoluções terapêuticas ${ }^{(3,4)}$ e diferentes graus de percepção, por parte dos sujeitos.

A impressão do paciente sobre sua saúde, como foco de análise, vem sendo estudada há algum tempo, objetivando a sua qualidade de vida, que pode ser afetada de inúmeras maneiras, de acordo com a saúde física, o estado psicológico, o nível de independência, as relações sociais e as crenças pessoais do indivíduo, além das características relevantes do seu meio ambiente ${ }^{(5)}$. Na avaliação da qualidade de vida, é imprescindível que a percepção do sujeito seja o foco do instrumento de análise. Desta forma, as principais ferramentas de verificação de consequências de um problema de saúde são os questionários de autoavaliação, para quantificar a percepção do indivíduo sobre o impacto de uma alteração em suas relações sociais, profissionais e financeiras ${ }^{(5)}$.

Avaliar a qualidade de vida relacionada à fonação e à deglutição demonstra o real impacto das alterações na vida dos indivíduos e é importante para a compreensão de como o paciente lida com as dificuldades, além de fornecer informações que poderão ser utilizadas para o melhor direcionamento do tratamento, enfocando-se os aspectos considerados relevantes para cada paciente em terapia ${ }^{(3)}$.

Os métodos tradicionais e objetivos de avaliação não mensuram precisamente os níveis de desvantagem e incapacidade de cada paciente. Os questionários que avaliam qualidade de vida e níveis de desvantagem proporcionam aos clínicos dados sobre o nível de intensidade da alteração e um panorama de como foi a intervenção terapêutica realizada, na visão do paciente ${ }^{(3)}$.

Alguns estudos sobre deglutição e disfagia têm monitorado a tosse ou mudanças na fonação como forma de desfecho, correlacionando achados vocais às informações clínicas e objetivas que evidenciam ou sugerem disfagia ${ }^{(6,7)}$. A videofluoroscopia da deglutição é, geralmente, considerada um método padrão de avaliação e diagnóstico de disfagia, por visualizar diretamente a laringe e trato aerodigestivo antes, durante e após a deglutição ${ }^{(8)}$.

Penetração e aspiração podem causar diversas mudanças de qualidade vocal ${ }^{(9)}$. Avaliar a voz do indivíduo disfágico pode trazer dados enriquecedores, principalmente pensando-se em triagens, por ser uma forma de avaliação rápida, simples e não invasiva, que pode ser realizada, inclusive, com a contribuição da análise acústica computadorizada ${ }^{(10)}$.

Durante a última década, estudos têm destacado a importância de analisar os sintomas vocais em conjunto a outros dados de impacto da disfonia e disfagia em câncer de cabeça e pescoço, sendo que associar informações de voz e sintomas aerodigestivos em um único instrumento oferece uma vantagem sobre os protocolos de autoavaliação que não investigam tais sintomas $^{(11-13)}$. Desta forma, para triagem de indivíduos disfônicos ou disfágicos, ou, ainda, para compor uma avaliação completa multidimensional, pode ser interessante olhar o indivíduo por uma perspectiva da autoavaliação de sintomas vocais e função glótica competente para queixas de voz e deglutição.

Este trabalho teve como objetivo avaliar a presença de sinais e sintomas vocais e função vocal em indivíduos com disfagia tratados de câncer de cabeça e pescoço e identificar características de eficiência e valores de corte dos protocolos que separam os indivíduos com queixas vocais dos vocalmente saudáveis.

\section{MÉTODOS}

O estudo foi aprovado pelo Comitê de Ética em Pesquisa em Seres Humanos da Fundação Antônio Prudente - Hospital do Câncer - A.C. Camargo/SP, em 18/6/2013, sob os pareceres números 1790/13 e 334.746 (CAAE: 17199613.3.0000.5432). Todos os sujeitos envolvidos assinaram o Termo de Consentimento Livre e Esclarecido - TCLE, assentindo, portanto, com a realização e divulgação desta pesquisa e seus resultados, conforme Resolução 466/12 (BRASIL. Resolução MS/CNS $n^{\circ} 466 / 12$ de 12 de dezembro de 2012).

Participaram deste estudo prospectivo 96 indivíduos, sendo 68 homens e 28 mulheres, com idade entre 28 e 87 anos (média de 58,82 anos), divididos em dois grupos: grupo experimental - GE e grupo controle - GC. O GE foi composto por pacientes tratados de câncer de cabeça e pescoço - CCP, com queixas de deglutição e diagnóstico de disfagia pela videofluoroscopia da deglutição, sendo esta a porta de entrada dos indivíduos deste grupo na pesquisa (estases na cavidade oral, orofaringe e/ou hipofaringe; penetração e/ou aspiração; disfagia orofaríngea indicada pela escala de gravidade da disfagia ${ }^{(14)}$, independente de sexo, idade, queixa vocal, tipo de tratamento ou sítio da lesão primária em câncer de cabeça e pescoço). O GC estudo foi composto de forma pareada ao GE, em média de idade e distribuição por sexo, sendo indivíduos vocalmente saudáveis e sem queixas e/ou alterações de deglutição e/ou voz, ou, ainda, histórico de câncer de cabeça e pescoço.

O GE foi constituído por 48 indivíduos, sendo 34 homens (média de idade 60,55 anos) e 14 mulheres (média de idade de 55,07 anos). O GC foi integrado por 48 indivíduos pareados por média de idade e sexo ao GE, sem queixas de deglutição e/ou voz autorrelatadas, sendo 34 homens (média de idade 60,29 anos) e 14 mulheres (média de idade 54,92 anos). Não houve diferenças na distribuição por sexo ( $p>0,99$, por meio do teste estatístico Qui-Quadrado) e média de idade ( $\mathrm{p}=0,44$, por meio do teste não paramétrico de Mann-Whitney) nos grupos GE e GC.

Nas informações demográficas, GE e GC se diferenciaram apenas nos quesitos tabagismo ( $\mathrm{p}=0,027$, por meio do teste Qui-Quadrado) e etilismo ( $\mathrm{p}<0,001$, por meio do teste Qui-Quadrado), com maiores ocorrências de não usuários no GC e ex-usuários no GE.

A distribuição demográfica das informações médicas, queixas de deglutição e vocais do grupo GE está descrita na Tabela 1.

Os pacientes elegíveis para o GE responderam a um questionário com dados de identificação e caracterização da amostra, Lista de Sinais e Sintomas Vocais - LSS e dois protocolos de autoavaliação vocal (Escala de Sintomas Vocais - ESV e Índice de Função Glótica - IFG). O questionário de identificação e caracterização da amostra foi composto por nome, data da avaliação, data de nascimento, idade, sexo, atividade profissional, informações de tabagismo, etilismo, medicamentos atuais e problemas de saúde, tipo e localização do tumor, tratamentos realizados (radioterapia, quimioterapia e/ou cirurgia), via de alimentação 
Tabela 1. Distribuição demográfica das informações médicas, queixas de deglutição e queixas vocais do grupo experimental $(\mathrm{N}=48)$

\begin{tabular}{|c|c|c|}
\hline Característica & Categoria & $\mathrm{N}(\%)$ \\
\hline \multirow{6}{*}{ Tipo de tumor } & Carcinoma espinocelular & $39(82)$ \\
\hline & Carcinoma papilífero & $4(8)$ \\
\hline & Carcinoma axial microcístico & $1(2)$ \\
\hline & Carcinoma indiferenciado & $2(4)$ \\
\hline & Carcinoma adenocístico & $1(2)$ \\
\hline & Osteosarcoma & $1(2)$ \\
\hline \multirow{6}{*}{ Local do tumor } & Cavidade Oral & $14(29)$ \\
\hline & Orofaringe & $17(35)$ \\
\hline & Hipofaringe & $1(2)$ \\
\hline & Laringe & $10(22)$ \\
\hline & Tireoide & $4(8)$ \\
\hline & Outros & $2(4)$ \\
\hline \multirow{2}{*}{ Tratamento: radioterapia } & Não & $15(31)$ \\
\hline & Sim & $33(69)$ \\
\hline \multirow{2}{*}{ Tratamento: quimioterapia } & Não & $29(60)$ \\
\hline & Sim & $19(40)$ \\
\hline \multirow{2}{*}{ Tratamento: cirurgia } & Não & $9(19)$ \\
\hline & Sim & $39(81)$ \\
\hline \multirow{3}{*}{ Alimentação enteral } & Não & $23(48)$ \\
\hline & Gastrostomia & $10(21)$ \\
\hline & Sonda nasoenteral & $15(31)$ \\
\hline \multirow{2}{*}{ Traqueostomia } & Não & $37(77)$ \\
\hline & Sim & $11(23)$ \\
\hline \multirow{2}{*}{ Queixa de deglutição } & Não & $0(0)$ \\
\hline & Sim & $48(100)$ \\
\hline \multirow{5}{*}{ Categorização das queixas de deglutição } & Engasgos & $18(38)$ \\
\hline & Dificuldades de deglutição & $13(27)$ \\
\hline & Sensação de alimento parado & $6(12)$ \\
\hline & Pigarro/tosse & $2(4)$ \\
\hline & Pedido médico/fonoaudiológico & $9(19)$ \\
\hline \multirow{2}{*}{ Queixa vocal } & Não & $0(0)$ \\
\hline & Sim & $48(100)$ \\
\hline \multirow{7}{*}{ Categorização das queixas vocais } & Voz grossa & $3(6)$ \\
\hline & Voz aguda & $2(4)$ \\
\hline & Perda de voz & $1(2)$ \\
\hline & Rouquidão & $7(15)$ \\
\hline & Voz diferente & $21(44)$ \\
\hline & Voz hipernasal & $9(19)$ \\
\hline & Voz fraca/baixa & $5(10)$ \\
\hline
\end{tabular}

Legenda: $\mathrm{N}=$ Número de sujeitos

(via oral, sonda nasoenteral - SNE, sonda nasogástrica - SNG ou gastrostomia), presença de traqueostomia, presença e categorização de queixas de deglutição, resultado da videofluoroscopia da deglutição (presença de estases na cavidade oral, orofaringe e/ou hipofaringe, penetração, aspiração, tipo e nível da disfagia), presença e categorização de queixas vocais e autoavaliação vocal (excelente, muito boa, boa, razoável ou ruim).

A Vocal Signs and Symptoms List - SSL ${ }^{(15)}$, traduzida e culturalmente adaptada para o português brasileiro como Lista de Sinais e Sintomas Vocais - LSS ${ }^{(16)}$ é um questionário simples e direto, com 14 sinais e sintomas relacionados à voz e ao trato aerodigestivo. A chave de respostas é apenas "sim" ou "não" para cada item, obtendo-se um número total de sinais e sintomas vocais autorrelatados, de zero (0) a 14.

O Glottal Function Index - GFI ${ }^{(17)}$, traduzido e culturalmente adaptado para o português brasileiro como Índice de Função Glótica - IFG ${ }^{(18)}$ é um protocolo de quatro itens, facilmente administrável, elaborado para avaliar a presença e o grau de disfunção vocal em adultos. O IFG possui uma chave de respostas (de "não é um problema" = zero (0) ponto a "é um problema muito grande" $=5$ pontos) e um escore total único, calculado pela soma das respostas das quatro questões do protocolo. Quanto maior o escore do IFG, maior a percepção do paciente sobre sua disfunção vocal.

A Voice Symptom Scale - VoiSS ${ }^{(1)}$, traduzida, culturalmente adaptada $^{(19)}$ e validada para o português brasileiro como Escala de Sintomas Vocais - ESV ${ }^{(20)}$ é um protocolo de 30 questões, divididas em três subescalas: 15 questões no domínio limitação, oito no emocional e sete no físico. A chave de respostas é composta por cinco itens: nunca $=$ zero $(0)$ ponto, raramente $=1$ ponto, às vezes $=2$ pontos, quase sempre $=3$ pontos e sempre $=4$ pontos. A ESV fornece quatro escores, a partir da soma bruta do valor assinalado em cada questão, sendo três parciais: limitação (soma dos resultados das questões $1,2,4,5,6,8,9,14,16,17,20$, $23,24,25$ e 27 , com valor de zero (0) a 60 pontos), emocional (soma dos resultados das questões 10,13,15, 18, 21, 28, 29 e 30, com valor de zero (0) a 32 pontos) e físico (soma das respostas das questões $3,7,11,12,19,22$ e 26, com valor de zero (0) a 
28 pontos) e um escore total (de zero (0) a 120 pontos (soma dos três escores parciais). Quanto maiores os escores da ESV, maior a percepção do paciente sobre seu desvio vocal.

Os indivíduos do GC responderam aos mesmos questionários e protocolos, com exceção das informações médicas e exame de videofluoroscopia da deglutição, não presentes neste grupo.

Por fim, o valor de corte que separou os indivíduos disfônicos pós-tratamento de câncer de cabeça e pescoço dos vocalmente saudáveis foi determinado com base nos indicadores de sensibilidade e especificidade, ambos utilizados em programas ou protocolos de triagem, por meio da Receiver Operating Characteristic-ROC curve. A curva ROC representa a relação entre a sensibilidade e especificidade de um teste, sendo um procedimento analítico simples para determinar o real valor do ponto de corte de um instrumento ${ }^{(21)}$.

$\mathrm{Na}$ análise estatística, o teste de Mann-Whitney foi utilizado para comparar os escores dos questionários e os valores de idade dos grupos experimental e controle. O teste Qui-Quadrado foi utilizado para comparar as distribuições das características clinicas/sociodemográficas entre os grupos. A curva ROC foi utilizada para determinar os valores de corte que melhor discriminassem os pacientes (GE x GC) em cada questionário. Para definir os valores de corte, foram considerados os maiores valores de sensibilidade e especificidade, simultaneamente. O nível de significância adotado foi de $5 \%$ e o software livre $\mathrm{R}$, versão 3.0.1 foi utilizado nas análises.

\section{RESULTADOS}

Na LSS, houve maior número médio de sinais e sintomas vocais para o GE, quando comparado ao GC (média de 1 sinal e sintoma vocal para o GC e 9 para o GE, p<0,001). Já no IFG, o GE apresentou maiores médias do escore total (20 pontos), quando comparado com o GC ( 0 ponto), evidenciando maior autopercepção da disfunção vocal. Na ESV, o GE apresentou maiores escores parciais e total (limitação $=23$, emocional $=5$, físico $=10$ e total $=39)$ que o $\mathrm{GC}($ limitação $=3$, emocional $=0$, físico $=2$ e total $=5$ ), com valor de $p<0,001$, mostrando que os indivíduos tratados de câncer de cabeça e pescoço e disfagia, mesmo que a queixa principal seja a deglutição, referem, em algum grau, prejuízo em suas vozes, como mostra a Tabela 2.

Com relação à autoavaliação vocal, os pacientes do GE apresentaram piores autorrelatos, classificando suas vozes apenas dentre as opções "ruim", "razoável" e "boa", quando comparados aos indivíduos do GC, que classificaram suas vozes dentre as opções "excelente", "muito boa" e "boa", como mostra a Tabela 3 .

Houve correlações positivas entre os escores totais dos protocolos IFG e ESV com a LSS. Portanto, quanto mais sinais e sintomas o paciente referiu, pior a sua percepção de disfunção vocal e maiores os prejuízos autorrelatados nos aspectos de limitação, emocionais e físicos da voz, embora as correlações não tenham sido fortes. Não houve correlações de idade com nenhum dos instrumentos de autoavaliação vocal, como mostra a Figura 1.

Os pacientes com sítio da lesão primária em orofaringe foram os que apresentaram mais sinais e sintomas vocais (média 9,5), seguidos pelos pacientes com sítio em laringe (média 8,4) e cavidade oral (média 7,8), com valor de $\mathrm{p}=0,05$. Os resultados da videofluoroscopia da deglutição dos indivíduos do GE encontram-se na Tabela 4.

Pacientes que apresentaram aspiração durante o exame de videofluoroscopia da deglutição tiveram, em média, piores valores (média 7,0) do escore emocional da ESV, quando comparados aos pacientes que não aspiraram (média 3,0), com

Tabela 2. Comparação dos escores dos protocolos e questionários de autoavaliação vocal no grupo experimental e no grupo controle

\begin{tabular}{|c|c|c|c|c|c|c|c|}
\hline Protocolos & Grupo & $\mathbf{N}$ & Média & Desvio padrão & Min-Máx & Mediana & Valor de $\mathrm{p}$ \\
\hline \multirow{2}{*}{ LSS } & $\mathrm{GC}$ & 48 & 1 & 1 & $0-2$ & 0,5 & \multirow{2}{*}{$<0,001^{*}$} \\
\hline & GE & 48 & 9 & 2 & $6-12$ & 8 & \\
\hline \multirow{2}{*}{ IFG } & $\mathrm{GC}$ & 48 & 0 & 1 & $0-2$ & 0 & \multirow{2}{*}{$<0,001^{*}$} \\
\hline & GE & 48 & 10 & 4 & $4-20$ & 9 & \\
\hline \multirow{2}{*}{ ESV - Limitação } & $\mathrm{GC}$ & 48 & 3 & 2 & $0-9$ & 2 & \multirow{2}{*}{$<0,001^{*}$} \\
\hline & GE & 48 & 23 & 12 & $0-54$ & 22,5 & \\
\hline \multirow{2}{*}{ ESV - Emocional } & $\mathrm{GC}$ & 48 & 0 & 0 & $0-2$ & 0 & \multirow{2}{*}{$<0,001^{*}$} \\
\hline & GE & 48 & 5 & 6 & $0-26$ & 4 & \\
\hline \multirow{2}{*}{ ESV - Físico } & $\mathrm{GC}$ & 48 & 2 & 2 & $0-8$ & 2 & \multirow{2}{*}{$<0,001^{*}$} \\
\hline & GE & 48 & 10 & 4 & $0-20$ & 10 & \\
\hline \multirow{2}{*}{ ESV - Total } & $\mathrm{GC}$ & 48 & 5 & 3 & $0-10$ & 5 & \multirow{2}{*}{$<0,001^{*}$} \\
\hline & GE & 48 & 39 & 17 & $17-89$ & 35 & \\
\hline
\end{tabular}

*Valores significantes ( $\mathrm{p} \leq 0,05)$ - Teste não paramétrico de Mann-Whitney

Legenda: GE = Grupo Experimental; GC = Grupo Controle; LSS = Lista de Sinais e Sintomas Vocais; IFG = Índice de Função Glótica; ESV = Escala de Sintomas Vocais; $\mathrm{N}=$ Número de sujeitos

Tabela 3. Autoavaliação vocal do grupo experimental $(N=48)$ e do grupo controle $(N=48)$

\begin{tabular}{|c|c|c|c|c|}
\hline \multirow{2}{*}{ Autoavaliação Vocal } & GC & GE & \multirow{2}{*}{ Total } & \multirow{2}{*}{ Valor de $p$} \\
\hline & N (\%) & N (\%) & & \\
\hline Excelente & 9 (19) & $0(0)$ & 9 & \\
\hline Muito boa & $28(58)$ & $0(0)$ & 28 & \\
\hline Boa & $11(23)$ & $8(17)$ & 19 & $<0,001^{*}$ \\
\hline Razoável & $0(0)$ & $28(58)$ & 28 & \\
\hline Ruim & $0(0)$ & $12(25)$ & 12 & \\
\hline
\end{tabular}

*Valores significantes $(p \leq 0,05)$ - Teste Qui-Quadrado

Legenda: $\mathrm{GE}=$ Grupo Experimental; GC = Grupo Controle; N = Número de sujeitos 


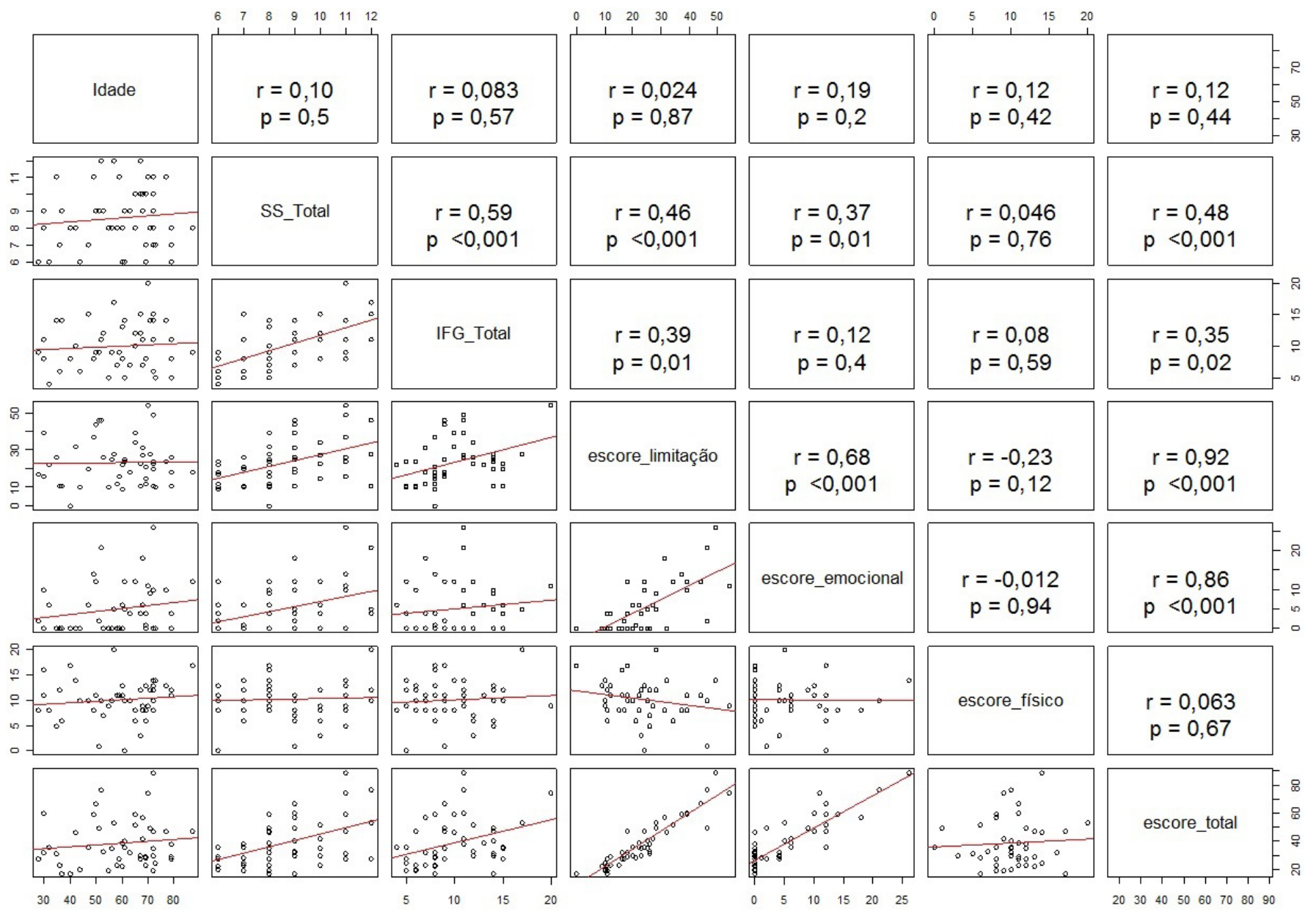

Figura 1. Matriz de correlação de média de idade, escore total da Lista de Sinais e Sintomas Vocais, escore total do Índice de Função Glótica e escores da Escala de Sintomas Vocais (Limitação, Emocional, Físico e Total) por meio do Teste de correlação de Pearson

Legenda: SS = Lista de Sinais e Sintomas Vocais; IFG = Índice de Função Glótica; escore_limitação = escore limitação do protocolo Escala de Sintomas Vocais; escore _ emocional = escore emocional do protocolo Escala de Sintomas Vocais; escore_físico = escore físico do protocolo Escala de Sintomas Vocais; escore_total = escore total do protocolo Escala de Sintomas Vocais

Tabela 4. Distribuição demográfica das informações da videofluoroscopia da deglutição do grupo experimental $(\mathrm{N}=48)$

\begin{tabular}{|c|c|c|}
\hline Característica & Categoria & $\mathrm{N}(\%)$ \\
\hline \multirow{2}{*}{ Estase: cavidade oral } & Não & $24(50)$ \\
\hline & Sim & $24(50)$ \\
\hline \multirow{2}{*}{ Estase: orofaringe } & Não & $4(8)$ \\
\hline & Sim & $44(92)$ \\
\hline \multirow{2}{*}{ Estase: hipofaringe } & Não & $14(29)$ \\
\hline & Sim & $34(71)$ \\
\hline \multirow{2}{*}{ Penetração } & Não & $10(21)$ \\
\hline & Sim & $38(79)$ \\
\hline \multirow{2}{*}{ Aspiração } & Não & $22(46)$ \\
\hline & Sim & $26(54)$ \\
\hline \multirow{2}{*}{ Conclusão da videofluoroscopia } & Deglutição funcional & $0(0)$ \\
\hline & Disfagia orofaríngea & $48(100)$ \\
\hline \multirow{6}{*}{ Nível da disfagia } & Deglutição normal & $0(0)$ \\
\hline & Disfagia discreta & $10(21)$ \\
\hline & Disfagia discreta/moderada & $17(35)$ \\
\hline & Disfagia moderada & $9(19)$ \\
\hline & Disfagia moderada/grave & $5(10)$ \\
\hline & Disfagia grave & $7(15)$ \\
\hline
\end{tabular}

Legenda: $\mathrm{N}$ = Número de sujeitos 
valor de $\mathrm{p}=0,04$. Dos pacientes que apresentaram estase na orofaringe, durante o exame de videofluoroscopia da deglutição $(\mathrm{N}=34)$, a maioria $(\mathrm{N}=31,78 \%$ ) autoavaliou sua voz como "razoável" ou "ruim", com valor de $p=0,0231$. Não houve diferenças significativas ou correlações entre os outros itens do questionário de identificação e caracterização da amostra e dados da videofluoroscopia da deglutição com os instrumentos de autoavaliação vocal utilizados.

Para a identificação dos valores de corte da LSS e dos escores totais dos protocolos IFG e ESV, que separam os indivíduos disfônicos com disfonia orgânica por câncer de cabeça e pescoço dos vocalmente saudáveis, os maiores valores de sensibilidade e especificidade foram considerados, como mostra a Tabela 5.

Os valores de área sob a curva, bem como os valores de sensibilidade e especificidade, foram iguais a 1, para os três questionários. Utilizando os valores de corte de 17 pontos para a ESV, 4 pontos para IFG e 6 pontos para a LSS, classificou-se corretamente $100 \%$ dos pacientes de câncer de cabeça e pescoço quanto às alterações vocais relacionadas a sinais e sintomas vocais e função glótica neste tipo específico de disfonia orgânica (Figura 2).

\section{DISCUSSÃO}

Indivíduos tratados de câncer de cabeça e pescoço comumente apresentam disfonia orgânica de diversos graus e impactos na qualidade de vida, principalmente quando os tumores são laríngeos ${ }^{(12,13,22,23)}$. As disfonias orgânicas podem gerar maior percepção de sintomas vocais relacionados ao prejuízo vocal, quando comparadas com outros tipos de disfonias, como as organofuncionais e funcionais ${ }^{(24)}$. Sabidamente, pacientes com alteração vocal frequentemente apresentam piores escores nos protocolos de autoavaliação vocal ${ }^{(17,20)}$, resultado esperado nos quadros de tumores laríngeos, nem sempre investigados nos casos de tumores de cabeça e pescoço, exceto laringe. Além disso, alterações sutis da qualidade vocal, ou, até mesmo, a conhecida voz molhada ${ }^{(6,7,10)}$, podem aparecer como primeiros sinais que indicam alguma função laríngea alterada, que, nas condições desses pacientes, podem ou não cursar com a disfagia.

A população experimental deste estudo não apresentava, como queixa principal, a alteração vocal; a participação no estudo deu-se em razão da queixa de deglutição, ou diagnóstico de disfagia na avaliação clínica, com indicação do exame de videofluoroscopia da deglutição que, em todos os casos da amostra do GE, comprovou a disfagia, no mínimo, de grau discreto. A disfagia é habitualmente apresentada durante e/ou após o tratamento de câncer de cabeça e pescoço, seja por mutilações anatômicas, como nas cirurgias, ou por radiação ou efeitos colaterais sistêmicos da quimioterapia, podendo impactar, de forma temporária ou definitiva, a alimentação por via oral desses indivíduos ${ }^{(25-27)}$. Porém, observou-se, ao longo da coleta, que os pacientes apresentavam queixas vocais após o tratamento do câncer de cabeça e pescoço, independente de serem tumores laríngeos ou de tireoide, em que se tem uma comprovada alteração vocal associada ao tratamento, quer seja cirurgia, quimioterapia e/ou radioterapia, esta última ocasionando mudanças histológicas da composição das pregas vocais, como fibrose e redução da

Tabela 5. Sensibilidade, especificidade e acurácia da curva ROC para os valores de corte da Lista de Sinais e Sintomas Vocais e dos escores totais dos protocolos Índice de Função Glótica e Escala de Sintomas Vocais

\begin{tabular}{|c|c|c|c|c|}
\hline & Valores de corte & Sensibilidade (\%) & Especificidade (\%) & Acurácia (\%) \\
\hline \multirow{7}{*}{ LSS } & $>12$ & 0 & 100 & 50 \\
\hline & $\ldots .$. & $\ldots$. & $\ldots .$. & $\ldots$. \\
\hline & 7 & 85 & 100 & 93 \\
\hline & $6^{*}$ & 100 & 100 & 100 \\
\hline & 2 & 100 & 83 & 92 \\
\hline & 1 & 100 & 50 & 75 \\
\hline & 0 & 100 & 0 & 50 \\
\hline \multirow{8}{*}{$\begin{array}{c}\text { IFG } \\
\text { escore total }\end{array}$} & $>20$ & 0 & 100 & 50 \\
\hline & 20 & 2 & 100 & 51 \\
\hline & $\ldots .$. & $\ldots .$. & $\ldots .$. & $\ldots .$. \\
\hline & 5 & 98 & 100 & 99 \\
\hline & $4^{\star}$ & 100 & 100 & 100 \\
\hline & 2 & 100 & 85 & 93 \\
\hline & 1 & 100 & 73 & 86 \\
\hline & 0 & 100 & 0 & 50 \\
\hline \multirow{9}{*}{$\begin{array}{c}\text { ESV } \\
\text { escore total }\end{array}$} & $>89$ & 0 & 100 & 50 \\
\hline & $\cdots \cdots$ & $\cdots \cdots$ & $\cdots \cdots$ & $\cdots$. \\
\hline & 19 & 96 & 100 & 98 \\
\hline & $17^{*}$ & 100 & 100 & 100 \\
\hline & 10 & 100 & 94 & 97 \\
\hline & ...... & $\ldots .$. & $\ldots .$. & $\ldots$. \\
\hline & 2 & 100 & 13 & 56 \\
\hline & 1 & 100 & 4 & 52 \\
\hline & 0 & 100 & 0 & 50 \\
\hline
\end{tabular}

Análise pela curva ROC. Em destaque: eficiência e valores de corte de cada instrumento

Legenda: LSS = Lista de Sinais e Sintomas Vocais; IFG = Índice de Função Glótica; ESV = Escala de Sintomas Vocais

*valores de corte da LSS, escore total do IFG e escore total da ESV com os mais altos valores de sensibilidade, especificidade e acurácia 
Curva ROC - Sinais e Sintomas

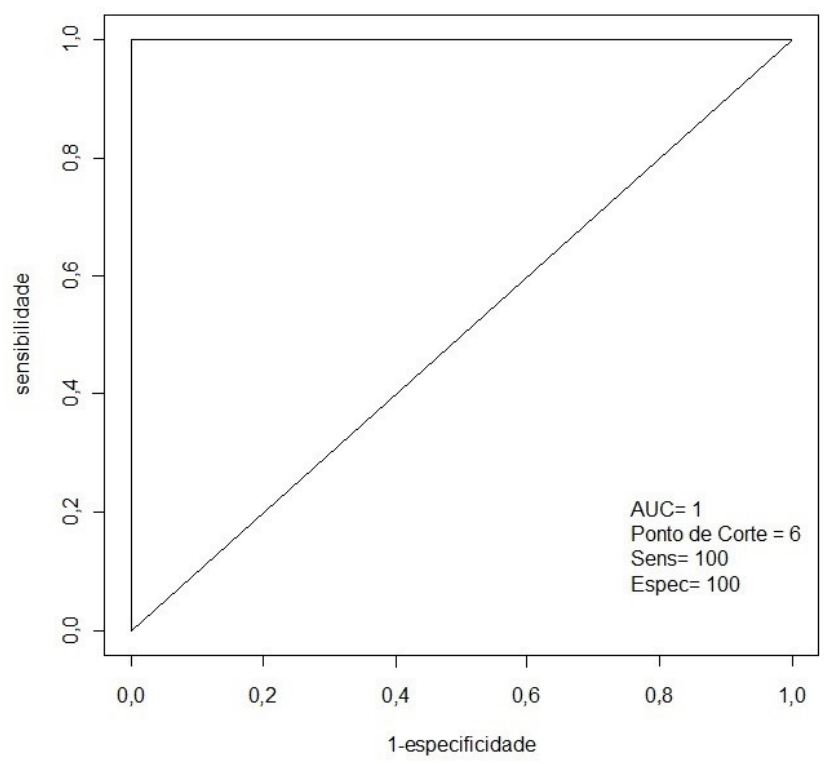

Curva ROC - IFG - Total

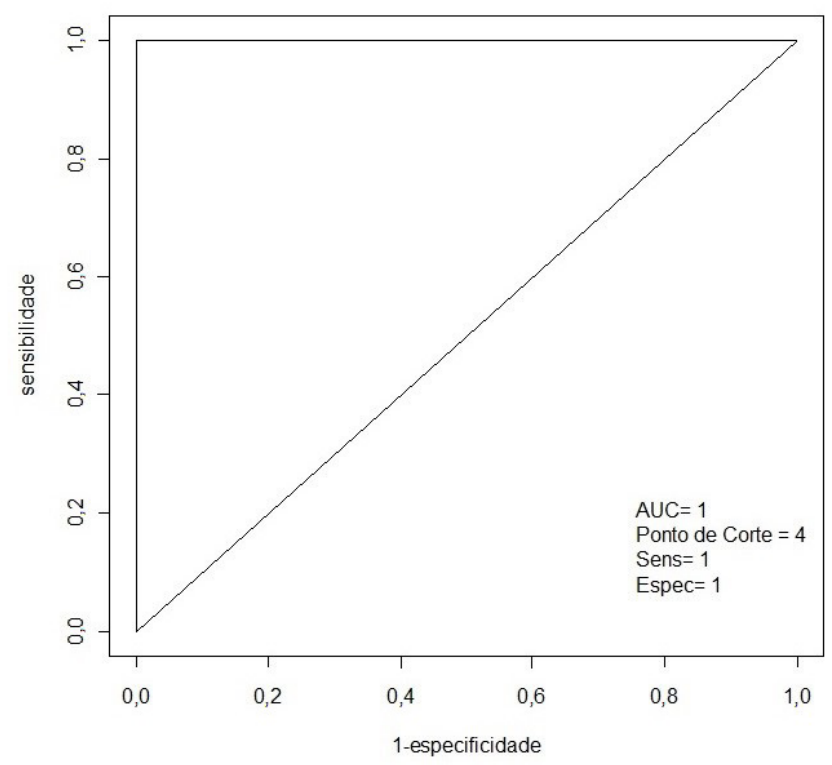

Curva ROC - ESV - Total

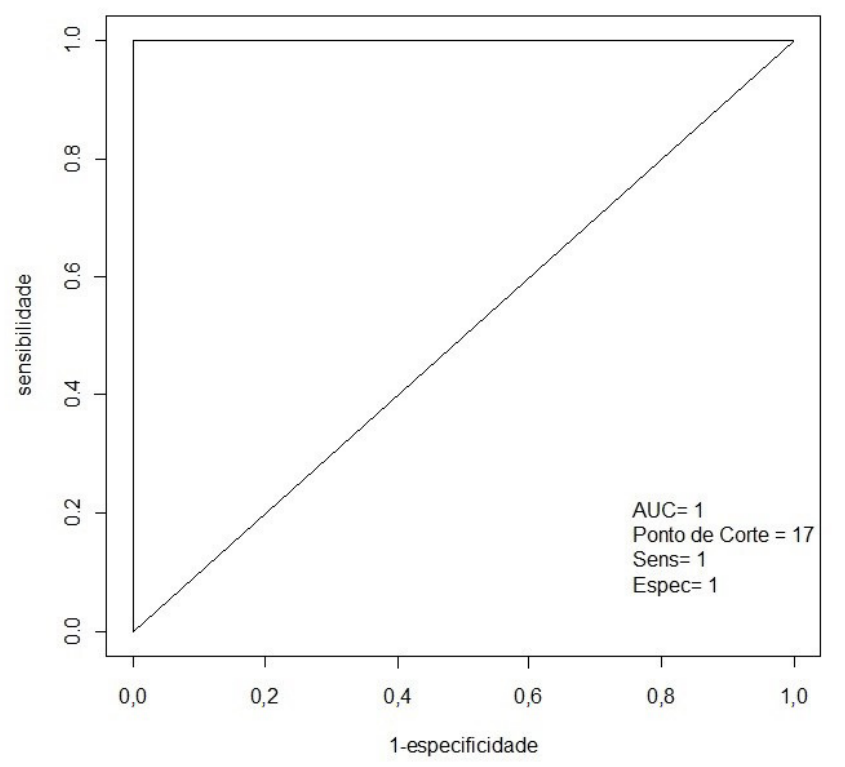

Figura 2. Áreas sob a curva ROC, valores de sensibilidade e especificidade e valores de corte da Lista de Sinais e Sintomas Vocais, do Índice de Função Glótica e da Escala de Sintomas Vocais

Legenda: IFG = Índice de Função Glótica; ESV = Escala de Sintomas Vocais; AUC = Área sob a curva; Sens = Sensibilidade; Espec = Especificidade

massa muscular ${ }^{(12,13,22,23,28)}$. Desta forma, analisar dados vocais do paciente disfágico pode trazer contribuições importantes e agregadoras para a avaliação fonoaudiológica, ainda mais por não se utilizar métodos invasivos para avaliação vocal ${ }^{(2,3,6,7,10)}$. Sendo assim, a análise dos dados vocais pode ser empregada em triagens, até mesmo para a elegibilidade de urgência em grandes populações ou serviços.

Estudos anteriores mostraram que indivíduos disfônicos apresentam, em média, mais sinais e sintomas vocais que os indivíduos vocalmente saudáveis ${ }^{(29)}$, pior autopercepção sobre a disfunção vocal ${ }^{(17)}$, maiores prejuízos vocais relacionados à limitação, aspectos emocionais e físicos da $\mathrm{voz}^{(20)}$ e pior autoavaliação vocal ${ }^{(20,24,29)}$, confirmando os achados deste estudo, como mostram as Tabelas 2 e 3. Pelo fato de os protocolos serem específicos, suas dimensões podem, de certo modo, ser intercambiáveis ${ }^{(24)}$, mesmo com correlações não tão fortes, pois cada protocolo possui sua particularidade de avaliação, como demonstrado na Figura 1.

O paciente tratado de tumor de cabeça e pescoço pode apresentar alterações de deglutição (Tabela 4), que podem ser transitórias ou permanentes, dependendo do tempo do tratamento realizado, bem como do tipo de tratamento, como radioterapia, quimioterapia, cirurgia ou estas modalidades de forma combinada ${ }^{(25-27)}$. As alterações de deglutição do paciente 
tratado de tumores de cabeça e pescoço podem, cada vez mais, cursar com alterações vocais, muitas vezes ao primeiro sinal/queixa do paciente, alertando o fonoaudiólogo sobre a necessidade de um acompanhamento minucioso desses indivíduos, tanto durante o tratamento oncológico combinado ${ }^{(25-27)}$, como após o tratamento, objetivando a reabilitação fonoaudiológica.

A LSS e os protocolos IFG e ESV mostraram-se como classificadores perfeitos na diferenciação de sujeitos com disfonias orgânicas por câncer de cabeça e pescoço e indivíduos vocalmente saudáveis: sensibilidade $100 \%$ (não permite falsos negativos) e especificidade 100\% (não permite falsos positivos), como mostram a Tabela 5 e a Figura 2 e já evidenciado em estudos anteriores, com disfônicos em geral, para a $\mathrm{ESV}^{(20,30)}$, sendo que, neste estudo, os valores de corte da ESV e do IFG para a população oncológica de câncer de cabeça e pescoço com queixas vocais foram muito próximos aos de outras pesquisas com os mesmos protocolos para disfônicos em geral ${ }^{(20,30)}$, podendo ser utilizados, com total propriedade, como instrumentos de triagem altamente confiáveis ao que se destinam avaliar, com contribuições importantes para a Fonoaudiologia, pois protocolos de autoavaliação são instrumentos rápidos de rastreio e, quando possuem valor de corte definidos para populações específicas, podem ser utilizados em triagens de grandes populações e de serviços com grandes demandas, principalmente os públicos.

\section{CONCLUSÃO}

Indivíduos tratados de câncer de cabeça e pescoço com disfagia apresentam mais sinais e sintomas vocais, pior percepção de sua disfunção vocal e maior prejuízo nos aspectos de limitação, emocionais e físicos da voz que indivíduos vocalmente saudáveis, independente de apresentarem queixa vocal como a principal. Os três instrumentos evidenciaram sensibilidade e especificidade máximas, podendo ser utilizados como recursos de triagem.

A Lista de Sinais e Sintomas Vocais - LSS, o Índice de Função Glótica - IFG e a Escala de Sintomas Vocais - ESV são classificadores perfeitos para avaliação da população específica oncológica de cabeça e pescoço, por apresentarem sensibilidade e especificidade máximas, com valores de corte definidos em 6 pontos para a SSV, 4 pontos para o IFG e 17 pontos para a ESV, sendo estes dois últimos com valores de corte para essa população muito próximos de outros estudos com disfônicos em geral.

\section{REFERÊNCIAS}

1. Mnari W, Kilani M, Harrathi K, Maatouk M, Koubaa J, Golli M. An unusual etiology of posttraumatic collet sicard syndrome: a case report. Pan Afr Med J. 2016;23:143. PMid:27279968.

2. Maffei C, Gonçalves MI, de Mello MM, Kluppel J Jr, Camargo PA. Pharyngeal cervical neurinoma: dysphonia and dysphagia. Braz J Otorhinolaryngol. 2007;73(5):718. http://dx.doi.org/10.1016/S18088694(15)30136-1. PMid:18094817.

3. Mangilli LD, Amoroso MR, Nishimoto IN, Barros AP, Carrara-deAngelis E. Voz, deglutição e qualidade de vida de pacientes com alteração de mobilidade de prega vocal unilateral pré e pós-fonoterapia. Rev Soc Bras Fonoaudiol. 2008;13(2):103-12. http://dx.doi.org/10.1590/ S1516-80342008000200003.
4. Fouquet ML, Vieira TP, Murata CJ, Gonçalves AJ. Efeito imediato da técnica de firmeza glótica nas laringectomias parciais horizontais supracricoides: estudo inicial. Rev Soc Bras Fonoaudiol. 2012;17(3):34650. http://dx.doi.org/10.1590/S1516-80342012000300018.

5. WHO: World Health Organization. The World Health Organization Quality of Life Instruments: the WHOQOL-100 and the WHOQOLBREF [Internet]. Geneva: WHO; 1997 [citado em 2016 Mai 2]. Disponível em: http://www.who.int/mental_health/media/68.pdf

6. Groves-Wright KJ, Boyce S, Kelchner L. Perception of wet vocal quality in identifying penetration/aspiration during swallowing. J Speech Lang Hear Res. 2010;53(3):620-32. http://dx.doi.org/10.1044/10924388(2009/08-0246). PMid:20029051.

7. Hey C, Lange BP, Aere C, Eberle S, Zaretsky Y, Sader R, Stöver T, Wagenblast J. Predictability of oral and laryngopharyngeal function for aspiration and limitation of oral intake in patients after surgery for head and neck cancer. Anticancer Res. 2013;33(8):3347-53. PMid:23898102.

8. Logemann JA. Approaches to management of disordered swallowing. Baillieres Clin Gastroenterol. 1991;5(2):269-80. http://dx.doi. org/10.1016/0950-3528(91)90030-5. PMid:1912652.

9. Groves-Wright KJ, Boyce S, Kelchner L. Perception of wet vocal quality in identifying penetration/aspiration during swallowing. J Speech Lang Hear Res. 2010;53(3):620-32. http://dx.doi.org/10.1044/10924388(2009/08-0246). PMid:20029051.

10. Kang YA, Kim J, Jee SJ, Jo CW, Koo BS. Detection of voice changes due to aspiration via acoustic voice analysis. Auris Nasus Larynx. 2018;45(4):801-6. http://dx.doi.org/10.1016/j.anl.2017.10.007. PMid:29097046.

11. Deary IJ, Wilson JA, Carding PN, MacKenzie K. VoiSS: a patientderived Voice Symptom Scale. J Psychosom Res. 2003;54(5):483-9. http://dx.doi.org/10.1016/S0022-3999(02)00469-5. PMid:12726906.

12. Loughran S, Calder N, MacGregor FB, Carding P, MacKenzie K. Quality of life and voice following endoscopic resection or radiotherapy for early glottic cancer. Clin Otolaryngol. 2005;30(1):42-7. http://dx.doi. org/10.1111/j.1365-2273.2004.00919.x. PMid:15748189.

13. Robertson SM, Yeo JC, Dunnet C, Young D, Mackenzie K. Voice, swallowing, and quality of life after total laryngectomy: results of the west of Scotland laryngectomy audit. Head Neck. 2012;34(1):59-65. http://dx.doi.org/10.1002/hed.21692. PMid:21416548.

14. O’Neil KH, Purdy M, Falk J, Gallo L. The dysphagia outcome and severity scale. Dysphagia. 1999;14(3):139-45. http://dx.doi.org/10.1007/ PL00009595. PMid:10341109.

15. Roy N, Merrill RM, Thibeault S, Gray SD, Smith EM. Voice disorders in teachers and the general population: effects on work performance, attendance, and future career choices. J Speech Lang Hear Res. 2004;47(3):542-51. http://dx.doi.org/10.1044/1092-4388(2004/042). PMid:15212567.

16. Behlau M, Zambon F, Guerrieri AC, Roy N. Epidemiology of voice disorders in teachers and nonteachers in Brazil: prevalence and adverse effects. J Voice. 2012;26(5):665.e9-18. http://dx.doi.org/10.1016/j. jvoice.2011.09.010. PMid:22516316.

17. Bach KK, Belafsky PC, Wasylik K, Postma GN, Koufman JA. Validity and reliability of the glottal function index. Arch Otolaryngol Head Neck Surg. 2005;131(11):961-4. http://dx.doi.org/10.1001/ archotol.131.11.961. PMid:16301366.

18. Madazio G, Zambon F, Moreti M, Vaiano T, Behlau M. Adaptação cultural e linguística da versão brasileira do Glottal Function Index - GFI. In: Anais do $21^{\circ}$ Congresso Brasileiro e $2^{\circ}$ Ibero-Americano 
de Fonoaudiologia; 2013 set 22-25; Porto de Galinhas. São Paulo: SBFa; 2013. 150-1.

19. Moreti F, Zambon F, Oliveira G, Behlau M. Cross-cultural adaptation of the Brazilian version of the Voice Symptom Scale: VoiSS. J Soc Bras Fonoaudiol. 2011;23(4):398-400. http://dx.doi.org/10.1590/ S2179-64912011000400018. PMid:22231064.

20. Moreti F, Zambon F, Oliveira G, Behlau M. Cross-cultural adaptation, validation, and cutoff values of the Brazilian version of the Voice Symptom Scale-VoiSS. J Voice. 2014;28(4):458-68. http://dx.doi. org/10.1016/j.jvoice.2013.11.009. PMid:24560004.

21. Martinez EZ, Louzada-Neto F, Pereira BB. A curva ROC para testes diagnósticos. Cad Saude Colet. 2003;11(1):7-31.

22. Bajaj Y, Uppal S, Sharma RK, Grace AR, Howard DM, Nicolaides AR, Coatesworth AP. Evaluation of voice and quality of life after transoral endoscopic laser resection of early glottic carcinoma. J Laryngol Otol. 2011;125(7):706-13. http://dx.doi.org/10.1017/S002221511100065X. PMid:21481298.

23. Robertson SM, Yeo JC, Sabey L, Young D, MacKenzie K. Effects of tumor staging and treatment modality on functional outcome and quality of life after treatment for laryngeal cancer. Head Neck. 2013;35(12):175963. http://dx.doi.org/10.1002/hed.23230. PMid:23483635.

24. Moreti F, Zambon F, Behlau M. Voice symptoms and vocal deviation self-assessment in different types of dysphonia. CoDAS. 2014;26(4):331-3. http://dx.doi.org/10.1590/2317-1782/201420130036. PMid:25211694.
25. Nguyen NP, Moltz CC, Frank C, Vos P, Smith HJ, Karlsson U, Nguyen LM, Rose S, Dutta S, Sallah S. Evolution of chronic dysphagia following treatment for head and neck cancer. Oral Oncol. 2006;42(4):374-80. http://dx.doi.org/10.1016/j.oraloncology.2005.09.003. PMid:16314138.

26. Nguyen NP, Frank C, Moltz CC, Karlsson U, Nguyen PD, Ward HW, Vos P, Smith HJ, Huang S, Nguyen LM, Lemanski C, Ludin A, Sallah S. Analysis of factors influencing Dysphagia severity following treatment of head and neck cancer. Anticancer Res. 2009;29(8):3299304. PMid:19661348.

27. Platteaux N, Dirix P, Dejaeger E, Nuyts S. Dysphagia in head and neck cancer patients treated with chemoradiotherapy. Dysphagia. 2010;25(2):139-52. http://dx.doi.org/10.1007/s00455-009-9247-7. PMid:19711127.

28. Tedla M, Valach M, Carrau RL, Varga I, Profant M, Mráz P, Weismann P. Impact of radiotherapy on laryngeal intrinsic muscles. Eur Arch Otorhinolaryngol. 2012;269(3):953-8. http://dx.doi.org/10.1007/ s00405-011-1686-8. PMid:21739096.

29. Oliveira G, Hirani SP, Epstein R, Yazigi L, Behlau M. Coping strategies in voice disorders of a Brazilian population. J Voice. 2012;26(2):20513. http://dx.doi.org/10.1016/j.jvoice.2010.10.023. PMid:21550778.

30. Behlau M, Madazio G, Moreti F, Oliveira G, Santos LM, Paulinelli $\mathrm{BR}$, Couto EB Jr et al. Efficiency and cutoff values of self-assessment instruments on the impact of a voice problem. J Voice. 2016;30(4):506. e9-18. http://dx.doi.org/10.1016/j.jvoice.2015.05.022. PMid:26168902. 